\title{
Structural changes during the switching transition of chalcogenide selector devices
}

Cite as: Appl. Phys. Lett. 115, 163503 (2019); https://doi.org/10.1063/1.5125215

Submitted: 21 August 2019. Accepted: 06 October 2019. Published Online: 15 October 2019

Yuzheng Guo, Huanglong Li, Wei Zhang ${ }^{(D)}$, and John Robertson
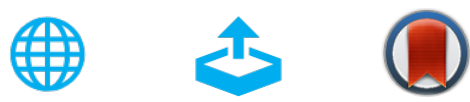

View Online

Export Citation

CrossMark

\section{ARTICLES YOU MAY BE INTERESTED IN}

One-step growth of wafer-scale monolayer tungsten disulfide via hydrogen sulfide assisted chemical vapor deposition

Applied Physics Letters 115, 163104 (2019); https://doi.org/10.1063/1.5123776

Ferroelectric properties of gradient doped $\mathrm{Y}_{2} \mathrm{O}_{3}: \mathrm{HfO}_{2}$ thin films grown by pulsed laser deposition

Applied Physics Letters 115, 162902 (2019); https://doi.org/10.1063/1.5121858

Nonvolatile memory based on redox-active ruthenium molecular monolayers

Applied Physics Letters 115, 162102 (2019); https://doi.org/10.1063/1.5108675

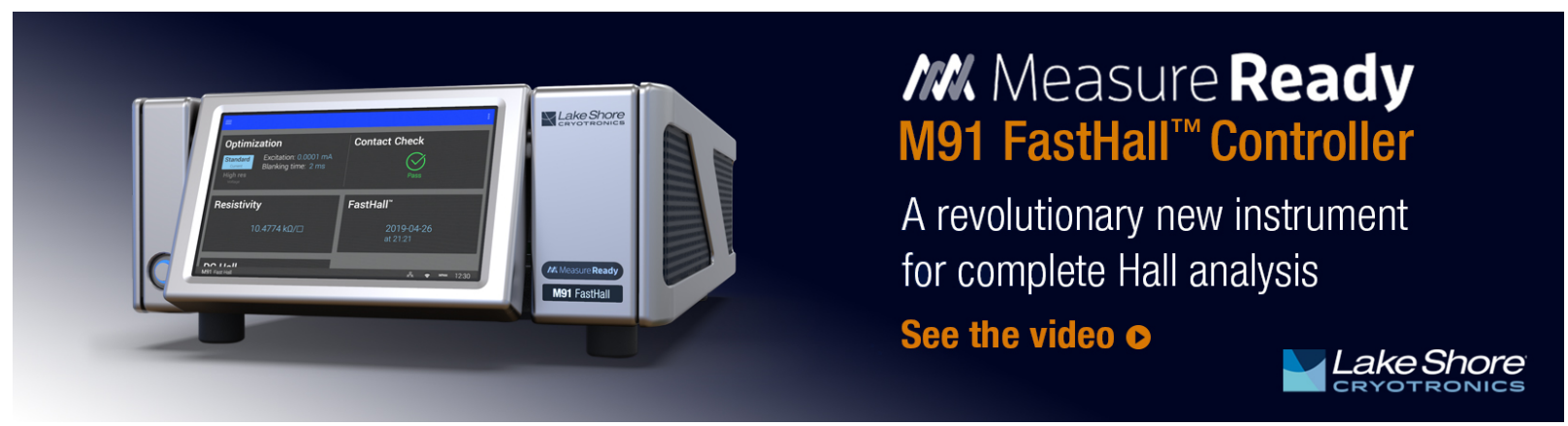




\title{
Structural changes during the switching transition of chalcogenide selector devices
}

\author{
Cite as: Appl. Phys. Lett. 115, 163503 (2019); doi: 10.1063/1.5125215 \\ Submitted: 21 August 2019 - Accepted: 6 October 2019 . \\ Published Online: 15 October 2019
}

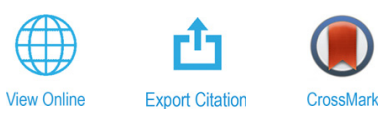

\author{
Yuzheng Guo, ${ }^{7}$ Huanglong Li, ${ }^{2}$ Wei Zhang, ${ }^{3}$ (D) and John Robertson ${ }^{4, a)}$
}

\begin{abstract}
AFFILIATIONS
'School of Electrical Engineering and Automation, Wuhan University, Wuhan, Hubei 43072, China

${ }^{2}$ Department of Precision Instrument, Tsinghua University, Beijing 100084, China

${ }^{3}$ Department of Electronics and Electrical Engineering, Liverpool John Moores University, Liverpool L3 3AF, United Kingdom

${ }^{4}$ Engineering Department, Cambridge University, Cambridge CB2 1PZ, United Kingdom
\end{abstract}

a) Author to whom correspondence should be addressed: jr@eng.cam.ac.uk

\begin{abstract}
Ovonic threshold switches are a favored choice for chalcogenide-based amorphous (a-) $\mathrm{GeSe}_{\mathrm{x}}$ selector devices used in cross-point arrays of nonvolatile memories. Previous models of their nonlinear high-field conduction proposed a largely electronic-only switching mechanism, within a fixed density of electronic states. Here, we use a density functional molecular-dynamics supercell calculation to show that the highcurrent excited state configuration of $\mathrm{a}-\mathrm{GeSe}_{\mathrm{x}}$ has structural changes such as additional Ge-Ge bonds and overcoordinated Ge sites, giving lower effective mass, more delocalized conduction states, and a lower ON resistance.
\end{abstract}

Published under license by AIP Publishing. https://doi.org/10.1063/1.5125215

There has recently been considerable interest in two-terminal nonlinear conduction elements to act as selector devices for nonvolatile memory devices arranged in XY cross-point arrays. ${ }^{1}$ The nonvolatile memories can be phase-change memories (PRAM) or resistive random access memories (RRAM) and are needed not only for storage class memory uses but also for neuromorphic computing and artificial intelligence applications. Some of the favored materials for selectors ${ }^{2-6}$ are the so-called Ovonic threshold switches (OTS). ${ }^{7,8}$ These are typically amorphous (a-) chalcogenides such as $\mathrm{a}-\mathrm{Ge}_{\mathrm{x}} \mathrm{Se}_{1-\mathrm{x}}$ semiconductor alloys. The OTS alloys have a similar composition to the phase change memory materials $(\mathrm{PCMs})^{9-13}$ but are typically selenides rather than tellurides as for PCMs. Functionally, the PCMs differ in that they undergo a rapid, nonvolatile structural phase transition between the low and high resistivity states of their crystalline and amorphous phases, respectively. ${ }^{9,10}$ On the other hand, the OTS materials undergo a rapid, volatile resistivity change which was generally assumed to be electronic in origin.

Adler et al. ${ }^{7}$ suggested this type of OTS mechanism. Building on this, Ielmini ${ }^{14}$ adapted a model of Poole-Frenkel high-field hopping in insulators such as $\mathrm{SiO}_{2}$ to show how a nonlinear conduction process could arise. This model created a negative differential resistance by the nonequilibrium occupation of localized tail states within an essentially fixed density of states (DOS), which implied a fixed underlying bonding network. However, transport data ${ }^{15}$ and density functional modeling ${ }^{5}$ showed that $\mathrm{a}-\mathrm{GeSe}_{\mathrm{x}}$ was a more typical semiconductor material than an insulator, with a moderate carrier mobility, no intermolecular tunnel barriers, and a calculated effective mass of $0.3 \mathrm{~m}_{\mathrm{e}}{ }^{16}$ Thus, the Ielmini model was modified into one suitable for semiconductors of moderate bandgap, using the ideas of quasi-Fermi levels. ${ }^{17}$

We initially took a-GeSe $e_{\mathrm{x}}$ consisting of a chemical ordered a$\mathrm{GeSe}_{2}$ network of heteropolar Ge-Se bonds which gave rise to the conduction band minimum (CBM) state. The nonlinear conduction process itself arose from some additional $\mathrm{Ge}-\mathrm{Ge}$ bonds which introduced localized gap states below the CBM. ${ }^{17}$

Recently, Chai et al. ${ }^{18}$ showed that the quasi-Fermi level model needed further modification. They observed that OTS selector devices undergo filamentary conduction in both their ON and OFF states, as in resistive RAM. They noted that once the OTS entered the ON state, when its current exceeded a threshold current, the device voltage was still less than in the OFF state, despite a many times higher current flow. Thus, the ON state conductance was too large to be consistent with hopping in localized tail states, and it would require conduction in extended states with a lower effective mass $\mathrm{m}^{*}$. Chai et al. ${ }^{18}$ proposed a state relaxation process to account for this. This implied that OTS switching was not a purely electronic process and involved some structural rearrangements, creating states of more delocalized character. Here, we show that the excited ON state configuration indeed 
differs from the ground state by the creation of more delocalized states, due to additional Ge-Ge bonds, and some overcoordinated Ge sites, which cause a lower $\mathrm{m}^{*}$.

In order to evaluate the amount of structural rearrangements involved in the high-field conductivity of OTS devices, we constructed various periodic supercell models of chemically ordered $\mathrm{Ge}_{40} \mathrm{Se}_{60}$ random networks, as previously. The networks are topologically disordered by a density functional molecular dynamics (MD) process. The calculations use the plane wave pseudopotential code CASTEP, ${ }^{19}$ with ultrasoft pseudopotentials and the Perdew, Burke, Ernzerhof-style generalized gradient approximation (GGA) for the exchange-correlation energy. The plane wave cut off energy is $340 \mathrm{eV}$. Only the $\Gamma$ point was used for the supercell calculation. Spin polarization is not included here. The initial structure was created by running the MD for 3 ps at $2000 \mathrm{~K}$ and then quenching a liquid sample from $1000 \mathrm{~K}$ to $300 \mathrm{~K}$ with isobaric-isothermal molecular dynamics at a quenching rate of $10 \mathrm{~K}$ per picosecond. The atomic positions are then fully relaxed, and the ground state is noted.

We then create an excited configuration by occupying the conduction band with two or four extra carriers. The excited state corresponds to where the semiconductor is held in its ON state above the threshold current. The CASTEP code used provides a compensated background charge for the extra electrons. An alternative would be an optical excitation generating unpaired electrons and holes, but the first method was computationally simpler. Molecular dynamics at $300 \mathrm{~K}$ is further performed, and then, the geometry was fully relaxed until the energy difference between three consecutive steps is below $10^{-3} \mathrm{eV}$ and the residual forces are below $0.03 \mathrm{eV} / \AA$.

A typical random network of $\mathrm{Ge}_{40} \mathrm{Se}_{60}$ of 480 atoms in the ground state is shown in Fig. 1. This is chemically ordered and has mainly fourfold coordinated Ge sites and twofold coordinated Se sites. The Ge excess above a stoichiometric $\mathrm{GeSe}_{2}$ composition and chemical disordering lead to $20 \%$ threefold Ge sites, $80 \%$ fourfold Ge sites, and $18 \%$ Ge-Ge bonds.

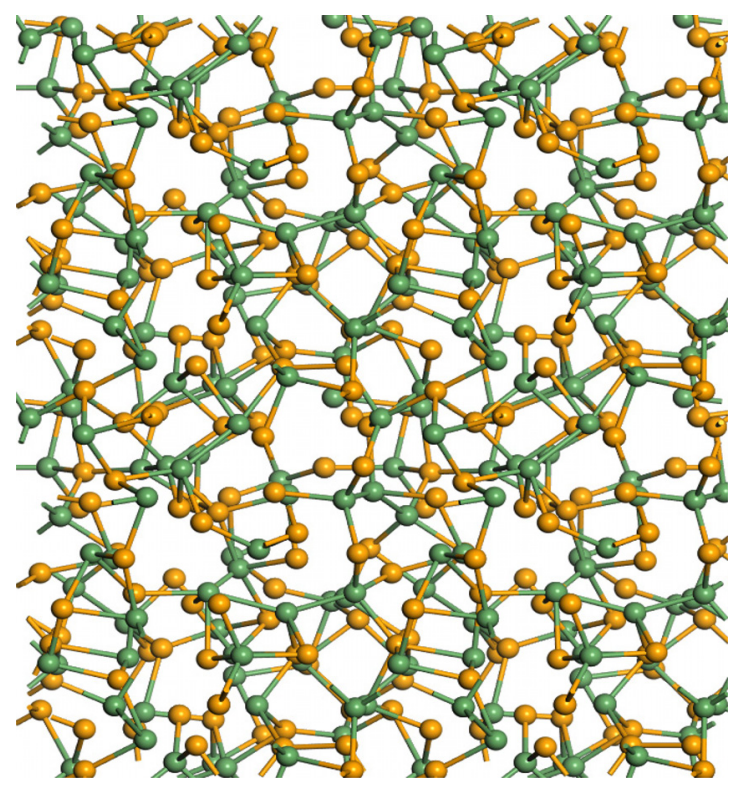

FIG. 1. Ground state of the 480 atom network. Green $=\mathrm{Ge}$ and orange $=\mathrm{Se}$.
We found earlier that the chemically ordered a-GeSe $e_{2}$ network has a bandgap of $\sim 1.5 \mathrm{eV}$ and that introducing an excess of $\mathrm{Ge}-\mathrm{Ge}$ bonds gives rise to states at or below the conduction band edge. ${ }^{5,16}$ Vertices of multiple Ge-Ge bonds give rise to a tail of localized states below the conduction band edge, which forms the basis of the nonlinear conduction mechanism.

Our networks differ from the more chemical ordered networks of $\mathrm{a}-\mathrm{Ge}_{1-\mathrm{x}} \mathrm{Se}_{\mathrm{x}}$ for $\mathrm{x}=0.67$ of Massobrio et al. ${ }^{20}$ and Micoulet et al. ${ }^{21}$ These authors generated networks by MD at $1000 \mathrm{~K}$, below the melting temperature of a- $\mathrm{Ge}_{2} \mathrm{Se}_{3}$, which disordered the network topology to represent bulk glasses. In our case, a higher initial temperature of $2000 \mathrm{~K}$ breaks network bonds and allows a mixture of fourfold and threefold Ge sites as in Ref. 22, which models deposited thin films.

Figure 2 shows an excited state for the 72 atom random network model. The bond connectivity map has been updated. Interestingly, although the atomic positions have not changed by much, some additional bonds have been formed. The new bonds are shown in blue in Fig. 2 and are found to be mainly Ge-Ge bonds.

Figure 3 shows a Ge partial radial distribution function (RDF) for the excited state in the 480 atom supercell. The first peak occurs at $2.45 \AA$ Á, which corresponds to direct Ge-Ge bonds for a tetrahedral bond angle as in pure Ge. The RDF shows a second peak at 2.7 Á. This is due to elongated Ge-Ge bonds and nontetrahedral sites. These consist mostly of threefold Ge sites that have been converted to fourfold Ge sites.

Figures 4(a) and 4(b) show some typical geometries of Ge sites in the excited state. Figure 4(a) shows a fourfold site derived from an octahedral geometry, with only 4 of the 6 bonds present. This is similar to the "octahedral" fourfold Ge site in GeSbTe alloys seen by Caravati et al. ${ }^{23,24}$ Figure 4 (b) shows a fivefold Ge site, also derived from a distorted octahedral Ge site. This fivefold Ge site resembles the fivefold Ge site found by Konstantiou et al. ${ }^{25}$ for defects in a GeSbTe alloy. Thus, some configurations in the excited state of GeSe resemble those in the ground state of GeTe.

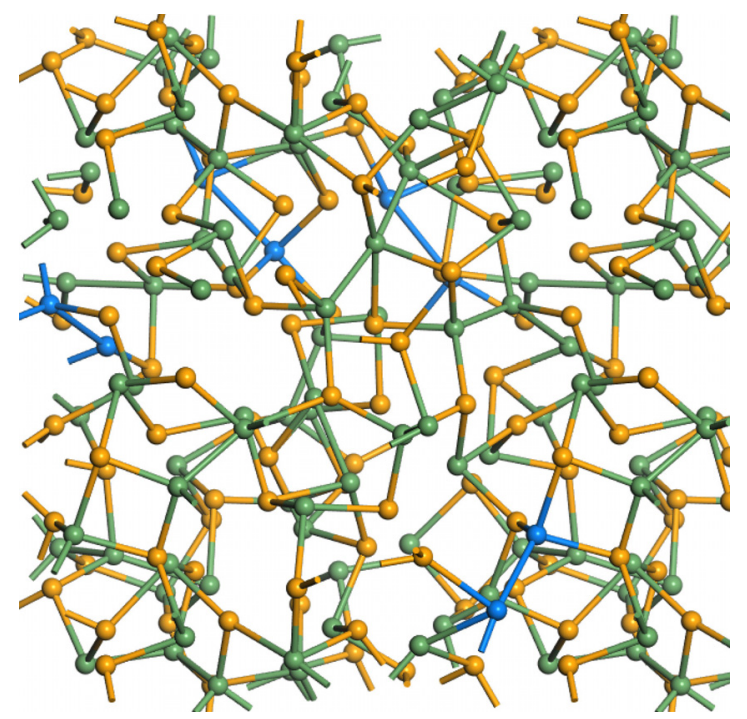

FIG. 2. Excited state of the 72 atom random network. Blue $=$ new bonds. 


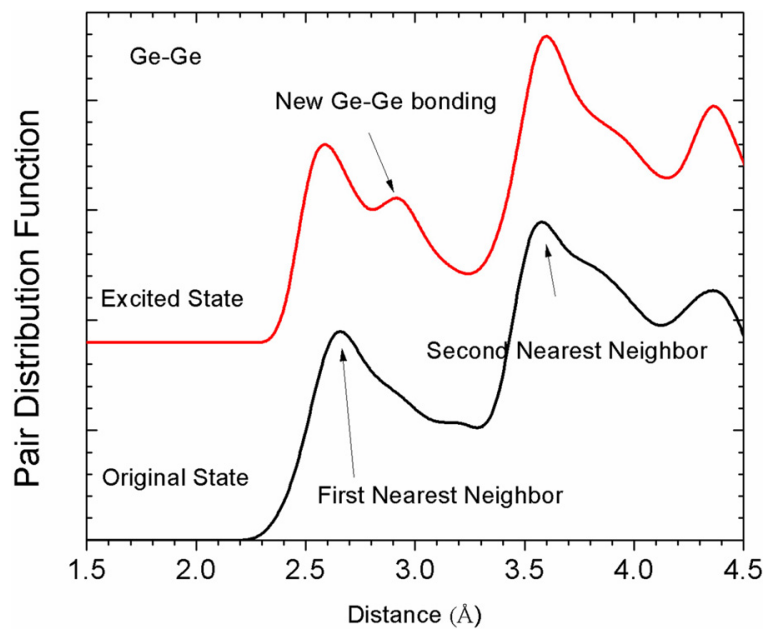

FIG. 3. Ge-Ge partial radial distribution function for the ground state and the excited state, showing extra Ge-Ge bonds at $2.70 \AA$ Á.

We then calculated the local density of states (DOS) for various networks (Fig. 5). Figure 5(a) compares the total DOS of the ground state and an excited state for one particular network. We see that the ground state has a local gap of $\sim 0.8 \mathrm{eV}$ with the Fermi level lying in midgap, while the excited state has a gap of $0.3 \mathrm{eV}$ with $\mathrm{E}_{\mathrm{F}}$ in the conduction band as a degenerate semiconductor. (There is no GGA error for this gap, as it is within the occupied states.) At the bottom of each panel, we show the inverse participation ratio (IPR) or localization of each energy level. There is a peak in IPR for the localized levels at the bottom of the conduction, as expected, and interestingly, the extra bonds wash this out in the excited state.

Figure 5(b) compares the partial DOS in an excited state of a typical threefold Ge site and one of the fourfold Ge sites, with a newly formed Ge-Ge bond. In some configurations, the partial density of states shows a gap state [Fig. 5(c)] whose wavefunction is a bonding state on one of the new elongated Ge-Ge bonds, Fig. 5(d). Thus, overall, the excited conducting state of the OTS GeSe based system with the additional Ge-Ge bonds has a much reduced bandgap with a lower $\mathrm{m}^{*}$ than the semiconducting ground state with $\mathrm{E}_{\mathrm{F}}$ in midgap, and this explains the low resistivity of this structure.

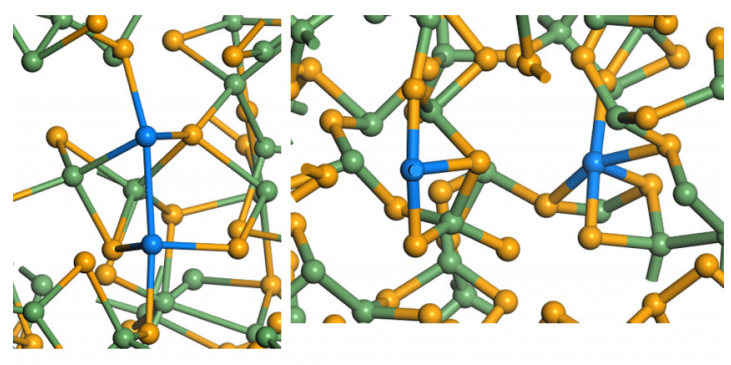

(a)

(b)

FIG. 4. (a) Showing the distorted octahedral coordination of Ge sites of a new Ge$\mathrm{Ge}$ bond (in blue). (b) Other miscoordinated Ge defect sites in the excited state (in blue).
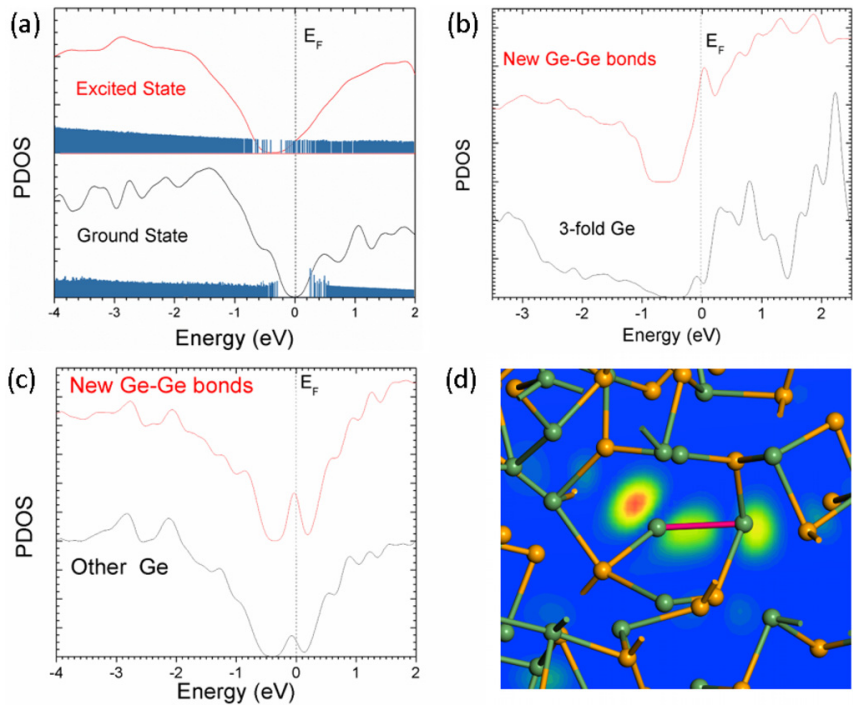

(d)

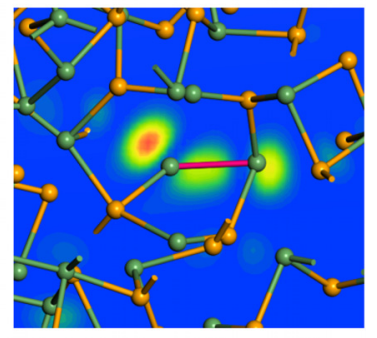

FIG. 5. (a) Comparison of total DOS for the ground and excited states of the 480 atom model. The inverse participation ratio (IPR) of the energy levels in each condition is shown at the bottom of the panels. (b) Partial density of states (PDOS) of a new Ge-Ge bond "defect" state and the adjacent Ge site in the excited state. (c) PDOS of a newly formed Ge defect bond, that is, forming a localized state below the conduction band edge. (d) Bonding state wavefunction of a new $\mathrm{Ge}-\mathrm{Ge}$ bond.

a-GeSe networks with 4:2 coordination have a similar network connectivity as $\mathrm{SiO}_{2}$ but with different bond angles for the group VI site. Similarly, a 3:3 coordinated GeSe network with $50 \%$ Ge will resemble orthorhombic GeSe, with trigonal Ge and Se sites. A formal charge transfer to give $\mathrm{Ge}^{-}-\mathrm{Se}^{+}$means that such trigonal sites obey the $8-\mathrm{N}$ bonding rule. Any Ge excess above a fraction of 33\% for a $4: 2$ coordinated $\mathrm{GeSe}_{2}$ network or $50 \%$ for a 3:3 coordinated GeSe network requires the presence of Ge-Ge bonds. On the other hand, alloy compositions between $33 \%$ and $50 \%$ Ge are possible by varying the fraction of four- and threefold Ge sites or by adding Ge-Ge bonds. For tellurides, Raty et al. ${ }^{22}$ related the resistance drift in GeTe phase change materials to a gradual change from 3:3 bonded GeTe toward a more 4:2 bonded GeTe with Ge-Ge bonding. The change from tellurides to selenides in OTS might be expected to favor more molecular bonding, a stronger adherence to the 8-N rule, and less overcoordination. From our calculations, we see that this is indeed true for the ground state, but the excited states of the network follow more overcoordinated bonding.

A general understanding of the nature of defects in these materials is important not only for selectors but also the key problem for the basic origin of resistance contrast in PRAMs as a whole. ${ }^{10}$ In crystalline Ge-Sb-Te (GST) or GeTe, the main defect is the Ge vacancy which pins the Fermi level near the valence band edge, ${ }^{26}$ giving the low ON state resistance. Defects observed by photomodulation spectroscopy of the amorphous phase pin the Fermi level near midgap, ${ }^{27,28}$ giving a high resistance OFF state. The nature of these defects has been only recently becoming clearer, ${ }^{25}$ and it must also be related to those of OTS materials.

A-Se is a typical covalently bonded amorphous chalcogenide. Its key defects are valence alternation pairs (VAP). ${ }^{29}$ VAPs consist of a negatively charged onefold coordinated site denoted $\mathrm{C}_{1}{ }^{-}$and a positively charged threefold coordinated site $\mathrm{C}_{3}{ }^{+}$, where $\mathrm{C}$ denotes chalcogen and the subscript denotes the coordination. The stability of VAPs 
was based on the idea that they conserved the number of bonds, so their creation energy would be relatively low, and thus, the VAP density would be high. Also, VAPs followed the $8-\mathrm{N}$ rule of bonding, when including all on-site electrons, including formal charge.

VAPs are likely to exist in a- $\mathrm{As}_{2} \mathrm{Se}_{3}$, as the basis of their photostructural changes. VAPs were proposed as possible defects in a-GST, ${ }^{30}$ but to date, in PCM tellurides, despite the observation of gap states, there is no strong evidence in favor of VAPs. ${ }^{31,32}$

In PCM tellurides, defect gap states have a complex structure and tend to consist of overcoordinated sites. ${ }^{24,33}$ The defects tend to be on cation sites and could consist of $\mathrm{Ge}_{5}{ }^{+}$and $\mathrm{Ge}_{3}{ }^{-}$sites. On the other hand, the defects in selenides in their ground states have more molecular bonding and tend to follow the $8-\mathrm{N}$ bonding rule. However, our calculations suggest that the defects of "excited states" of a-GeSe follow those of telluride PCMs with overcoordinated sites.

In summary, we have calculated the excited state configuration of amorphous GeSe alloy Ovonic threshold switches, with electronic occupation of the conduction band, using density function molecular dynamics. We find that these states undergo a mild structural transition with the formation of additional Ge-Ge bonds mainly between previously threefold coordinated Ge sites. This leads to overcoordinated distorted octahedral Ge sites, and a delocalization of conduction states, which accounts for the high conductivity of the ON state. Thus, the previously proposed electronic only transition using fixed density of states to explain the nonlinear resistivity of OTS materials needs modification to include the effect of varying density of states and state delocalization due to mild structural transitions.

We acknowledge the funding from the EC H2020 project Phase change switch.

\section{REFERENCES}

${ }^{1}$ H. S. P. Wong, S. Raoux, S. Kim, J. Liang, and K. E. Goodson, Proc. IEEE 98, 2201 (2010).

${ }^{2}$ B. Govoreanu, G. L. Donadio, K. Opsomer, W. Devulder, V. V. Afanasev, T. Witter, S. Clima, N. S. Avasarala, A. Redolfi, S. Kundu, O. Richard, D. Tsvetanova, G. Pourtois, C. Detaverniert, L. Goux, and G. S. Kar, in Technical Digest of VLSI Technology (2017), p. T72.

${ }^{3}$ G. Liu, L. Wu, and Z. Song, Appl. Phys. Lett. 111, 252102 (2017).

${ }^{4}$ T. Kim, H. Choi, and M. Kim, in Technical Digest of IEDM (2018).

${ }^{5}$ S. Clima, B. Govoreanu, K. Opsomer, A. Velea, N. S. Avasarala, W. Devulder, I. Shlyakhov, G. L. Donadio, T. Witter, S. Kundu, L. Goux, V. Afanasiev, G. S. Kar, and G. Pourtois, in Technical Digest of IEDM (2017), p. 4.1.
${ }^{6}$ A. Verdy, M. Bernard, J. Garrione, G. Bourgeois, M. C. Cryille, E. Nolot, N. Castellani, P. Noe, C. Socquet-Clerc, T. Magis, G. Sassine, G. Molas, G. Navarro, and E. Nowak, in Technical Digest of IEM (2018), p. 37.4.

${ }^{7}$ D. Adler, M. S. Shur, M. Silver, and S. R. Ovshinsky, J. Appl. Phys. 51, 3289 (1980).

${ }^{8}$ A. E. Owen, J. M. Robertson, and C. Main, J. Non-Cryst. Solids 32, 29 (1979).

${ }^{9}$ S. Raoux, W. Welnic, and D. Ielmini, Chem. Rev. 110, 240 (2010).

${ }^{10}$ D. Lencer, M. Salinga, and M. Wuttig, Adv. Mater. 23, 2030 (2011).

${ }^{1}$ A. V. Kolobov, P. Fons, A. I. Frenkel, A. L. Ankudinov, J. Tominaga, and T. Uruga, Nat. Mater. 3, 703-708 (2004).

${ }^{12}$ K. Shportko, S. Kremers, M. Woda, D. Lencer, J. Robertson, and M. Wuttig, Nat. Mater. 7, 653 (2008).

${ }^{13}$ B. Huang and J. Robertson, Phys. Rev. B 81, 081204(R) (2010).

${ }^{14}$ D. Ielmini, Phys, Rev. B 78, 035308 (2008).

${ }^{15}$ G. I. Kim and J. Shirafuji, Jpn. J. Appl. Phys., Part 1 17, 1789 (1978).

${ }^{16} \mathrm{H}$. Li and J. Robertson, Microelectron. Eng. 216, 111037 (2019).

${ }^{17} \mathrm{H}$. Li and J. Robertson, Sci. Rep. 9, 1867 (2019).

${ }^{18}$ Z. Chai, W. Zhang, R. DeGraeve, S. Clima, F. Hatem, J. F. Zhang, P. Freitas, and J. Marsland, in Technical Digest of VLSI (2019), p. T18.5.

${ }^{19}$ S. J. Clark, M. D. Segall, C. J. Pickard, P. J. Hasnip, M. J. Probert, K. Refson, and M. C. Payne, Z. Kristallogr. 220, 567 (2005).

${ }^{20}$ C. Massobrio, P. S. Salmon, R. A. Martin, M. Micoulet, and A. Pasquarello, Phys. Rev. B 79, 174201 (2009); S. Le Roux, A. Bouzid, M. Boero, and C. Massobrio, ibid. 86, 224201 (2012).

${ }^{21}$ M. Micoulet, A. Kachmar, M. Bauchy, S. Le Roux, C. Massobrio, and M. Boero, Phys. Rev. B 88, 054203 (2013).

${ }^{22}$ J. Y. Raty, W. Zhang, J. Luckas, C. Chen, R. Mazzarello, C. Bichara, and M. Wuttig, Nat. Commun. 6, 7467 (2015); J. Y. Raty, Phys. Status Solidi RRL 131, 800590 (2019).

${ }^{23}$ S. Caravati, M. Bernasconi, T. D. Kuhne, M. Krack, and M. Parrinello, Appl. Phys. Lett. 91, 171906 (2007).

${ }^{24}$ S. Caravati, M. Bernasconi, and M. Parrinello, J. Phys.: Condens. Matter 21, 255501 (2009).

${ }^{25}$ K. Konstantiou, F. C. Mocanu, T. H. Lee, and S. R. Elliott, Nat. Commun. 10, 3065 (2019).

${ }^{26}$ A. H. Edwards, A. C. Pineda, P. A. Schultz, M. G. Martin, A. P. Thomson, and H. P. Hjalmarson, Phys. Rev. B 73, 045210 (2006).

${ }^{27}$ C. Longeaud, J. Luckas, D. Krebs, R. Carius, J. Klomfass, and M. Wuttig, J. Appl. Phys. 112, 113714 (2012).

${ }^{28}$ J. Luckas, D. Krebs, S. Grothe, J. Klomfass, R. Carius, and M. Wuttig, J. Mater. Res. 28, 1139 (2013).

${ }^{29}$ M. Kastner, D. Adler, and H. Fritzsche, Phys. Rev. Lett. 37, 1504 (1976).

${ }^{30}$ A. Pirovano, A. L. Lacita, A. Benvenuti, F. Pellizer, and R. Bez, IEEE Trans. Electron Device 51, 452 (2004).

${ }^{31} \mathrm{~B}$. Huang and J. Robertson, Phys. Rev. B 85, 125305 (2012).

${ }^{32} \mathrm{X}$. Yu and J. Robertson, Can. J. Phys. 92, 671 (2014).

${ }^{33}$ J. Y. Raty, in Technical Digest, EPCOS Conference, Grenoble, France, September (2019). 